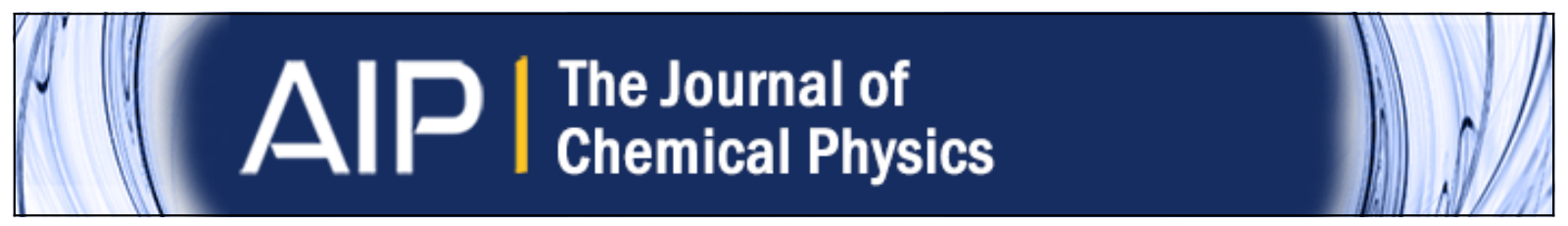

\title{
A hybrid perturbed-chain SAFT density functional theory for representing fluid behavior in nanopores
}

Gulou Shen, Xiaoyan Ji, and Xiaohua Lu

Citation: The Journal of Chemical Physics 138, 224706 (2013); doi: 10.1063/1.4808160

View online: http://dx.doi.org/10.1063/1.4808160

View Table of Contents: http://scitation.aip.org/content/aip/journal/jcp/138/22?ver=pdfcov

Published by the AIP Publishing

\section{Articles you may be interested in}

Comparison of several classical density functional theories for the adsorption of flexible chain molecules into cylindrical nanopores

J. Chem. Phys. 139, 234902 (2013); 10.1063/1.4843655

A hybrid perturbed-chain SAFT density functional theory for representing fluid behavior in nanopores: Mixtures J. Chem. Phys. 139, 194705 (2013); 10.1063/1.4825078

Electric double layer capacitance of restricted primitive model for an ionic fluid in slit-like nanopores: A density functional approach

J. Chem. Phys. 137, 234705 (2012); 10.1063/1.4771919

Fourier space approach to the classical density functional theory for multi-Yukawa and square-well fluids

J. Chem. Phys. 137, 104104 (2012); 10.1063/1.4749381

Density-functional theory for polymer-carbon dioxide mixtures: A perturbed-chain SAFT approach

J. Chem. Phys. 137, 054902 (2012); 10.1063/1.4742346

\section{AlIP Re-register for Table of Content Alerts}




\title{
A hybrid perturbed-chain SAFT density functional theory for representing fluid behavior in nanopores
}

\author{
Gulou Shen, ${ }^{1,2}$ Xiaoyan $\mathrm{Ji}^{2}$ and Xiaohua $\mathrm{Lu}^{1, \mathrm{a})}$ \\ ${ }^{1}$ State Key Laboratory of Materials-Oriented Chemical Engineering, Nanjing University of Technology, \\ 5 Xinmofan Road, Nanjing 210009, People's Republic of China \\ ${ }^{2}$ Division of Energy Science/Energy Engineering, Luleå University of Technology, 97187 Luleå, Sweden
}

(Received 12 February 2013; accepted 7 May 2013; published online 13 June 2013)

\begin{abstract}
A hybrid statistical mechanical model, which is fully consistent with the bulk perturbed-chain statistical associating fluid theory (PC-SAFT) in describing properties of fluids, was developed by coupling density functional theory with PC-SAFT for the description of the inhomogeneous behavior of real chain molecules in nanopores. In the developed model, the modified fundamental measure theory was used for the hard sphere contribution; the dispersion free energy functional was represented with weighted density approximation by averaging the density in the range of interaction, and the chain free energy functional from interfacial statistical associating fluid theory was used to account for the chain connectivity. Molecular simulation results of the density profile were compared with model prediction, and the considerable agreement reveals the reliability of the proposed model in representing the confined behaviors of chain molecules in an attractive slit. The developed model was further used to represent the adsorptions of methane and carbon dioxide on activated carbons, in which methane and carbon dioxide were modeled as chain molecules with the parameters taken from the bulk PC-SAFT, while the parameters of solid surface were obtained from the fitting of gas adsorption isotherms measured experimentally. The results show that the model can reliably reproduce the confined behaviors of physically existing substances in nanopores.

(C) 2013 AIP Publishing LLC. [http://dx.doi.org/10.1063/1.4808160]
\end{abstract}

\section{INTRODUCTION}

Understanding of fluid behaviors in nanopores and nanochannels is of considerable importance in various industrial processes and scientific fields such as adsorption, wetting, lubrication, and tribology. ${ }^{1,2}$ Due to wall-molecule interactions and wall geometry the confined fluid in nanopores or nanochannels is inhomogeneous and shows quite different characteristics compared to their behavior in bulk. A number of experimental measurements, molecular simulations, and theoretical models were used to study the properties of confined fluid in nanoslit or nanotube. ${ }^{3-6}$ Among them, the classical density functional theory (DFT) is an efficient theoretical method to study the phase behavior and thermodynamic properties of inhomogeneous fluids.

In the last two decades, several DFT models have been developed to predict the properties of inhomogeneous simple atomic and complex fluids. A significant progress was the development of DFT models for representing the properties of polyatomic fluid on the basis of Wertheim's first order thermodynamic perturbation theory. ${ }^{7-11}$ Compared to molecular simulation, most such DFT models accurately predict the microstructure of inhomogeneous fluid, but it is infeasible to use such models to represent properties of real substances due to the fact that most of these models were applied for model molecules with integer segment numbers. On the other hand, the bulk properties of fluids have been extensively studied in

\footnotetext{
a)Electronic mail: xhlu@njut.edu.cn.
}

which the segment number is not an integer in most cases. Thus, it will be very desirable to develop a DFT model representing the behavior of real substances with one set of molecular parameters for both inhomogeneous and bulk cases.

A reliable and robust theory for representing the properties of bulk fluid is a prerequisite for such a DFT model development. Statistical associating fluid theory (SAFT) equation of state (EoS) is one of the most promising approaches describing phase behavior of bulk fluids. SAFT was originally developed by Chapman et al. ${ }^{12}$ on the basis of Wertheim's first order thermodynamic perturbation theory. Several versions of SAFT have been proposed later, such as SAFT-VR, ${ }^{13}$ soft-SAFT, ${ }^{14}$ SAFT $1,{ }^{15}$ SAFT2,${ }^{16,17}$ and PC-SAFT, ${ }^{18,19}$ and widely used for systems containing non-polar, polar, ionic, and associating substances including gases, solvents, homopolymers, copolymers, and electrolytes. Details of theories and applications can be found in reviews. ${ }^{20-22}$

Based on SAFT EoS, several groups have developed DFT models and further used them to describe the phase behaviors of mixture as well as interfacial tensions. Gloor et $_{\text {al. }}{ }^{23-25} \mathrm{de}-$ veloped a DFT model on the basis of SAFT-VR with a local density approximation, and the model accurately predicts the interfacial tension. Based on perturbed-chain polar statistical associating fluid theory (PCP-SAFT), Gross ${ }^{26}$ proposed a DFT reproducing the surface tension of non-polar and polar substances. The free energy functional for dispersion attraction is expressed as a first order perturbation term. As the functional is not consistent with bulk PC-SAFT, a local density approximation was used to compensate the difference. In 
addition, both of these two DFTs were only used to represent the interfacial properties where the external field is weak. For confined fluids where the external field plays an important role, Tripathi and Chapman ${ }^{9}$ developed an accurate DFT for the confined hard chain fluid, but the dispersive interaction was not considered in their model. Therefore, we can conclude that there is no consistent model available to represent the fluid behavior in nanopores and bulk phase simultaneously for real substances.

In this work, a hybrid PC-SAFT-DFT model, completely consistent with bulk PC-SAFT, will be developed to represent the fluid behavior in nanopores. PC-SAFT was chosen because of its well-known performance for bulk fluids. The developed model will be used to predict the density profile and the prediction will be compared with molecular simulation results for model verification. Moreover, the model will be used to represent the experimental adsorption isotherms of methane and carbon dioxide on activated carbon to illustrate the model performance for real (physically existing) substances instead of model molecules.

\section{THEORY}

The model developed in this work was based on PCSAFT in which the hard-chain, dispersive, and associative interactions were considered. There are two versions of PCSAFT, one is for square-well (SW) chain molecule (first version $)^{18}$ and the other is for modified square-well chain molecule (second version). ${ }^{19}$ The only difference of these two models is on the universal constants in dispersion term and the hard sphere diameter, i.e., the temperature-dependent segment diameter was used in the second version of the PC-SAFT model, while in the first version of PC-SAFT, and the segment diameter was a constant. The details of PC-SAFT can be found in the work by Gross and Sadowski. ${ }^{18,19}$ In this work, the DFT model development was based on PC-SAFT (second version), ${ }^{19}$ while the developed model can be easily extended to the first version of PC-SAFT.

In DFT, for an open system, the grand potential $\Omega$ at fixed $\mu, \mathrm{V}$, and $\mathrm{T}$ is given by the equation,

$$
\Omega[\rho(\boldsymbol{r})]=A[\rho(\boldsymbol{r})]-\int d r^{\prime} \rho\left(\boldsymbol{r}^{\prime}\right)\left(\mu-m V_{\text {ext }}\left(\boldsymbol{r}^{\prime}\right)\right),
$$

where $A$ is the Helmholtz free energy, $\rho(\boldsymbol{r})$ is the molecular density of fluid, $\mu$ is the chemical potential, $m$ is the number of segments in a chain, and $V_{\text {ext }}(\boldsymbol{r})$ is the external field acting on the segment.

Following PC-SAFT EoS, the Helmholtz free energy $A$ can be expressed as

$$
\begin{aligned}
A[\rho(\boldsymbol{r})]= & A^{i d}[\rho(\boldsymbol{r})]+A^{h s}[\rho(\boldsymbol{r})]+A^{\text {chain }}[\rho(\boldsymbol{r})] \\
& +A^{d i s p}[\rho(\boldsymbol{r})]+A^{\text {assoc }}[\rho(\boldsymbol{r})],
\end{aligned}
$$

where $A^{\text {id }}$ is the ideal free energy, $A^{\text {hs }}, A^{\text {chain }}, A^{\text {disp }}$, and $A^{\text {assoc }}$ are the excess free energies due to hard sphere, chain, dispersive, and associative interactions, respectively. The extension of PC-SAFT to inhomogeneous fluid is described in the following part.

\section{A. Ideal and hard chain terms}

Based on Wertheim's thermodynamics perturbation theory, Tripathi and Chapman ${ }^{9,27}$ derived the free energy functional by considering the chain molecule as a mixture of associating monomers, and the chain was formed in the limit of complete association. The developed model is consistent with the bulk SAFT model. In this work, the model developed by Tripathi and Chapman ${ }^{9,27}$ was used to represent the ideal and chain terms. Following this approach, the reference ideal free energy is written as the free energy of the ideal atomic gas mixture,

$$
\beta A^{i d}[\rho]=\int d \boldsymbol{r} m \rho(\boldsymbol{r})(\ln \rho(\boldsymbol{r})-1),
$$

where $\beta=1 / k T$ and $k$ is the Boltzmann constant. The de Broglie wavelength was ignored in Eq. (3). The hard sphere free energy can be calculated using the modified fundamental measure theory, ${ }^{28}$ which is based on the equation for the hard sphere developed by Boublik ${ }^{29}$ and Mansoori et al. ${ }^{30}$ and is compatible with PC-SAFT. The hard sphere free energy is given by

$$
\beta A^{h s}[\rho]=\int d \boldsymbol{r} \Phi^{h s}\left[n_{\alpha}(\boldsymbol{r})\right]
$$

with

$$
\begin{aligned}
\Phi^{h s}\left[n_{\alpha}(\boldsymbol{r})\right]= & -n_{0} \ln \left(1-n_{3}\right)+\frac{n_{1} n_{2}-\boldsymbol{n}_{v 1} \cdot \boldsymbol{n}_{v 2}}{1-n_{3}} \\
& +\frac{1}{36 \pi}\left[n_{3} \ln \left(1-n_{3}\right)+\frac{n_{3}^{2}}{\left(1-n_{3}\right)^{2}}\right] \\
& \times \frac{n_{2}^{3}-3 n_{2} \boldsymbol{n}_{v 2} \cdot \boldsymbol{n}_{v 2}}{n_{3}^{3}} .
\end{aligned}
$$

In Eqs. (4) and (5), $n_{\alpha}(\boldsymbol{r})$ are weighted densities of Rosenfeld, ${ }^{31}$

$$
n_{\alpha}(\boldsymbol{r})=m \int d \boldsymbol{r}^{\prime} \rho\left(\boldsymbol{r}^{\prime}\right) w^{\alpha}\left(\boldsymbol{r}-\boldsymbol{r}^{\prime}\right)
$$

where $w^{\alpha}$ represents weight functions, i.e., four scalar and two vector functions,

$$
\begin{aligned}
w^{2}(\boldsymbol{r}) & =\delta(d / 2-r), w^{3}(\boldsymbol{r})=\theta(d / 2-r), \\
\boldsymbol{w}^{v 2}(\boldsymbol{r}) & =\boldsymbol{r} / r \delta(d / 2-r), \\
w^{0}(\boldsymbol{r}) & =w^{2}(\boldsymbol{r}) / \pi d^{2}, w^{1}(\boldsymbol{r})=w^{2}(\boldsymbol{r}) / 2 \pi d, \\
\boldsymbol{w}^{v 1}(\boldsymbol{r}) & =\boldsymbol{w}^{v 2}(\boldsymbol{r}) / 2 \pi d .
\end{aligned}
$$

In Eq. (7), $\delta(r)$ and $\theta(r)$ are the Dirac delta and Heaviside step functions, respectively, and $d$ is the hard sphere diameter. In PC-SAFT, Barker and Henderson's perturbation theory was used, i.e., the temperature-dependent segment diameter was calculated with $d(T)=\sigma\left[1-0.12 \exp \left(-3 \varepsilon_{f} / k T\right)\right]$, where $\varepsilon_{f}$ is the energy parameter of fluid-fluid interaction and $\sigma$ is the segment diameter. 
Following the approach by Tripathi and Chapman, ${ }^{9,27}$ the chain free energy functional is given by

$$
\begin{aligned}
\beta A^{\text {chain }}[\rho] & \\
= & -(m-1) \int d \boldsymbol{r} \rho(\boldsymbol{r}) \\
& \times\left[\ln \left\{y^{\text {cont }}[\bar{\rho}(\boldsymbol{r}), d] \int d \boldsymbol{r}^{\prime} \frac{\delta\left(\left|\boldsymbol{r}-\boldsymbol{r}^{\prime}\right|-d\right)}{4 \pi d^{2}} \rho\left(\boldsymbol{r}^{\prime}\right)\right\}-1\right],
\end{aligned}
$$

where $y^{\text {cont }}[\bar{\rho}(\boldsymbol{r}), d]$ is the value of cavity correlation function at contact and it is given by

$$
y^{\text {cont }}[\bar{\rho}(\boldsymbol{r}), d]=\frac{1}{1-\bar{\zeta}_{3}}+\frac{3 d \bar{\zeta}_{2}}{2\left(1-\bar{\zeta}_{3}\right)^{2}}+\frac{d^{2}\left(\bar{\zeta}_{2}\right)^{2}}{2\left(1-\bar{\zeta}_{3}\right)^{3}}
$$

and $\bar{\zeta}_{i}=\frac{\pi}{6} m d^{i} \bar{\rho}(\boldsymbol{r})$, which is evaluated at the "coarse-grained density," i.e.,

$$
\bar{\rho}(\boldsymbol{r})=\frac{3}{4 \pi d^{3}} \int_{\left|\boldsymbol{r}-\boldsymbol{r}^{\prime}\right|<d} d \boldsymbol{r}^{\prime} \rho\left(\boldsymbol{r}^{\prime}\right) .
$$

\section{B. Dispersion term}

A weighted density approximation can be used to construct the dispersion free energy functional. ${ }^{32-35}$ Following this approximation the dispersion free energy functional is given by

$$
\beta A^{d i s p}[\rho]=\int d \boldsymbol{r} \rho(\boldsymbol{r}) f\left(\bar{\rho}_{d i s p}(\boldsymbol{r})\right),
$$

where

$$
\begin{aligned}
f\left(\bar{\rho}_{d i s p}(\boldsymbol{r})\right)= & -12.0 m \frac{\varepsilon}{k T}\left(\frac{\sigma}{d}\right)^{3} \bar{\eta}_{d i s p}(\boldsymbol{r}) I_{1} \\
& -6.0 m^{2}\left(\frac{\varepsilon}{k T}\right)^{2}\left(\frac{\sigma}{d}\right)^{3} C_{1}\left(\bar{\eta}_{d i s p}(\boldsymbol{r})\right) \bar{\eta}_{d i s p}(\boldsymbol{r}) I_{2},
\end{aligned}
$$

with

$$
\begin{aligned}
& C_{1}\left(\bar{\eta}_{d i s p}(\boldsymbol{r})\right) \\
& =1+m \frac{8 \bar{\eta}_{d i s p}(\boldsymbol{r})-2 \bar{\eta}_{d i s p}(\boldsymbol{r})^{2}}{\left(1-\bar{\eta}_{d i s p}(\boldsymbol{r})\right)^{4}} \\
& +(1-m) \frac{20 \bar{\eta}_{d i s p}(\boldsymbol{r})-27 \bar{\eta}_{d i s p}(\boldsymbol{r})^{2}+12 \bar{\eta}_{d i s p}(\boldsymbol{r})^{3}-2 \bar{\eta}_{d i s p}(\boldsymbol{r})^{4}}{\left[\left(1-\bar{\eta}_{d i s p}(\boldsymbol{r})\right)\left(2-\bar{\eta}_{d i s p}(\boldsymbol{r})\right)\right]^{2}} .
\end{aligned}
$$

In Eqs. (11) and (12a), $\bar{\eta}_{\text {disp }}(\boldsymbol{r})$ is the average packing fraction $\left(=\frac{\pi}{6} m d^{3} \bar{\rho}_{d i s p}(r)\right)$ in which the weighted density is given by

$$
\bar{\rho}_{d i s p}(\boldsymbol{r})=\int d \boldsymbol{r}^{\prime} \rho\left(\boldsymbol{r}^{\prime}\right) w_{d i s p}\left(\boldsymbol{r}-\boldsymbol{r}^{\prime}\right),
$$

where $w_{\text {disp }}(\boldsymbol{r})$ is the weighting function.

As the model developed in this work is based on PCSAFT, and the square-well potential was used in PC-SAFT version 1 and the modified square-well potential was used in
PC-SAFT version 2, we assumed that the potential of PCSAFT in both versions to be square-well in the DFT model development.

The weighting functions have been studied extensively, ${ }^{32-35}$ and a comprehensive introduction of the weighted density approximation can be found in the review of Zhou. ${ }^{36} \mathrm{~A}$ common normalized mean field weighting function is defined as $w_{d i s p}(\boldsymbol{r})=u_{a t t r}(\boldsymbol{r}) / \int d \boldsymbol{r} u_{a t t r}(\boldsymbol{r})$, where $u_{a t t r}(\boldsymbol{r})$ is the attractive potential. This method has been used to obtain the weighted density for Lennard-Jones (LJ) fluids. ${ }^{32,33}$ Following the definition, the weighted density for square-well fluid can also be obtained by integrating the density in the range of attraction. Meanwhile, Ye et al. ${ }^{10}$ proposed another approximate method, and the weighted density was calculated by integrating the density in the range of interaction, i.e., $w_{d i s p}(\boldsymbol{r})=\frac{3 \theta(\lambda \sigma-r)}{4 \pi(\lambda \sigma)^{3}}$. It has been systematically proved that this approximate method could provide satisfactory density profiles of square-well chain confined in a slit. ${ }^{10}$ Therefore, in this work, the method proposed by Ye et al. ${ }^{10}$ was used for the DFT model based on PC-SAFT, i.e., the weighted density is calculated by

$$
\bar{\rho}_{d i s p}(\boldsymbol{r})=\frac{3}{4 \pi(\lambda \sigma)^{3}} \int_{\left|\boldsymbol{r}-\boldsymbol{r}^{\prime}\right|<\lambda \sigma} d \boldsymbol{r}^{\prime} \rho\left(\boldsymbol{r}^{\prime}\right),
$$

where $\lambda \sigma$ represents the weighting distance.

\section{Association term}

For association term, $\mathrm{Yu}$ and $\mathrm{Wu}^{7}$ proposed an approach for a mixture of associating hard spheres. We used the same approach in this work. The free energy functional due to association is given by

$$
\beta A^{a s s o c}[\rho]=\int d \boldsymbol{r} \Phi^{a s s o c}\left[n_{\alpha}(\boldsymbol{r})\right],
$$

where

$$
\begin{aligned}
\Phi^{a s s o c}\left[n_{\alpha}(\boldsymbol{r})\right]= & \sum_{j=1}^{s} S^{j}\left(\frac{n_{2}^{2}-\boldsymbol{n}_{v 2} \cdot \boldsymbol{n}_{v 2}}{\pi d^{2} n_{2}}\right) \\
& \times\left[\ln X^{j}(\boldsymbol{r})-\frac{X^{j}(\boldsymbol{r})}{2}+\frac{1}{2}\right] .
\end{aligned}
$$

In Eq. (15), $s$ is the number of types of association sites, $S^{j}$ is the number of association sites of type $\mathrm{j}, n_{\alpha}(\boldsymbol{r})$ are the weighted densities calculated with Eq. (7), and $X^{j}$ is the fraction of molecules not bonded at site $j$ and calculated using the mass action equation,

$$
X^{j}=\frac{1}{1+\sum_{l=1, l \neq j}^{s} s^{l}\left(\frac{n_{2}^{2}-\boldsymbol{n}_{v 2} \cdot \boldsymbol{n}_{v 2}}{\pi d^{2} n_{2}}\right) X^{l}(\boldsymbol{r}) \Delta^{j l}(\boldsymbol{r})} .
$$




\section{Numerical procedure}

Minimization of the grand potential with respect to the density profile yields the following Euler-Lagrange:

$$
\frac{\delta \Omega[\rho(\boldsymbol{r})]}{\delta \rho(\boldsymbol{r})}=\frac{\delta A[\rho(\boldsymbol{r})]}{\delta \rho(\boldsymbol{r})}-\left(\mu-m V_{\text {ext }}(\boldsymbol{r})\right)=0 .
$$

At equilibrium, $\mu$ is equal to the bulk chemical potential $\mu_{b}$ that is calculated from the temperature and pressure for pure components. The equilibrium density profile can be solved using simple Picard iteration.

In planar geometry, the three-dimensional integral can be reduced to a one-dimensional integral. In planar geometry, the detailed expressions of functional derivative integrals of hard sphere and chain terms can be referred to Gross's work. ${ }^{26}$ The derivatives of $X^{j}$ with respect to density were calculated using the generalized procedure proposed by Tan et al. ${ }^{37}$ Derivatives were formulated as linear simultaneous equations and then written in a matrix form. The method solving the linear equations and the corresponding code were given in the literature of Tan et al. ${ }^{37}$

\section{RESULTS AND DISCUSSION}

To verify the model, the developed model was used to predict the density profile in different cases, and the model prediction results were compared with molecular simulation results. To illustrate the model performance for real (physically existing) substances besides "model molecules," furthermore, the model was used to represent the adsorption isotherms of methane and carbon dioxide on activated carbons, in which the parameters describing the external force were obtained from the fitting of the experimental adsorption data.

As mentioned in the foregoing text, there are two versions of PC-SAFT, and the DFT model developed in this work is based on the second version of PC-SAFT. However, most of the results obtained in the molecular simulation were obtained with the assumption of square-well fluids with $\lambda=1.5$, which is the case of the first version of PC-SAFT. Therefore, in the part of model verification, in order to compare the DFT model predictions with molecular simulation results, the DFT model developed in this work was extended to the first version of PC-SAFT by replacing the temperature-dependent segment diameter with a fixed segment diameter. In addition, in the second version of PC-SAFT, the potential of fluid is a modified square-well potential, which approaches to the LennardJones attraction, and subsequently, the DFT model prediction in this work was also compared with LJ fluid.

To calculate the weighted density using Eq. (13b), the square-well length $\lambda$ for fluids needs to be determined. In the comparison with molecule simulation (Secs. III A and III B), the value of $\lambda$ was taken from the literature for square-well fluids and equals to 1.5. However, $\lambda$ was set to be 1.5 in Eq. (13b) in order to investigate the compatibility of the developed PC-SAFT-DFT with LJ fluids (Sec. III C). In the part of the model representation of gas adsorption isotherm for real substances (Sec. III D), $\lambda$ was also set to be 1.5.
TABLE I. Summary of the cases used for prediction of the density profiles of square-well fluids in square-well slit pores.

\begin{tabular}{lcc}
\hline \hline$k T / \varepsilon_{f}$ & $\rho_{a v} \sigma^{3}$ & No. case \\
\hline 1.0 & 0.689 & 1 \\
& 0.727 & 2 \\
& 0.811 & 3 \\
1.2 & 0.606 & 4 \\
& 0.667 & 5 \\
& 0.778 & 6 \\
\hline \hline
\end{tabular}

In the DFT model developed in this work, the interaction between surface and fluid molecules was represented with external forces, and it can be square-well, LJ or even more complicated interactions. In the comparison with molecule simulation, the interaction between surface and fluid was taken from the literature.

In the part of the model representation of gas adsorption isotherm for real substances, the developed model on the basis of the second version of PC-SAFT was used, and LJ potential was used to describe the interaction between surface and fluid.

\section{A. Density profiles of square well fluids in square-well slit pores}

The density profile of square well fluid $(m=1)$ in slit pores with square well potential has been simulated by others at different temperatures and densities, which is summarized in Table I. In Table I, $\rho_{a v}$ is the average density in the pore (= $\frac{1}{H} \int_{0}^{H} d z \rho(z), H$ is the width of the slit). The developed DFT model was used to predict the density profile by considering the square-well interaction between surface and fluid as an external force, as shown in Eq. (18),

$$
V_{e x t}(z)=\left\{\begin{array}{cl}
\infty & z<0 \\
-\varepsilon_{w} & z<\lambda_{w} \sigma \\
0 & z>\lambda_{w} \sigma
\end{array}\right.
$$

where $z$ is the perpendicular distance between fluid and surface.

In modeling, the fluid-surface, fluid-fluid interaction parameters were taken from literatures directly as listed. In all cases, $m=1, H=9 \sigma, \varepsilon_{w} / k T=1.5, \lambda=1.5$, and $\lambda_{\mathrm{w}}=0.5$. The density profile of fluids under all conditions listed in Table I was predicted and compared with Monte Carlo (MC) simulation results. ${ }^{38}$ The comparison shows that the model prediction agrees extremely well with the MC simulation results. Figure 1 illustrates the comparison results of cases 3 and 6 in Table $I$ in order to show the good agreements, and the density profile of case 6 was shifted upward for clarity.

\section{B. Density profiles of square-well chain fluids in square-well slit pores}

The developed model was used to predict the density profile of square-well chain fluids $(m>1)$ in a slit pore with a square-well interaction between surface and fluid represented as external force as shown in Eq. (18). In modeling, the average packing fraction was defined by $\eta=\frac{\pi}{6} m \sigma^{3} \rho_{a v}$ where 


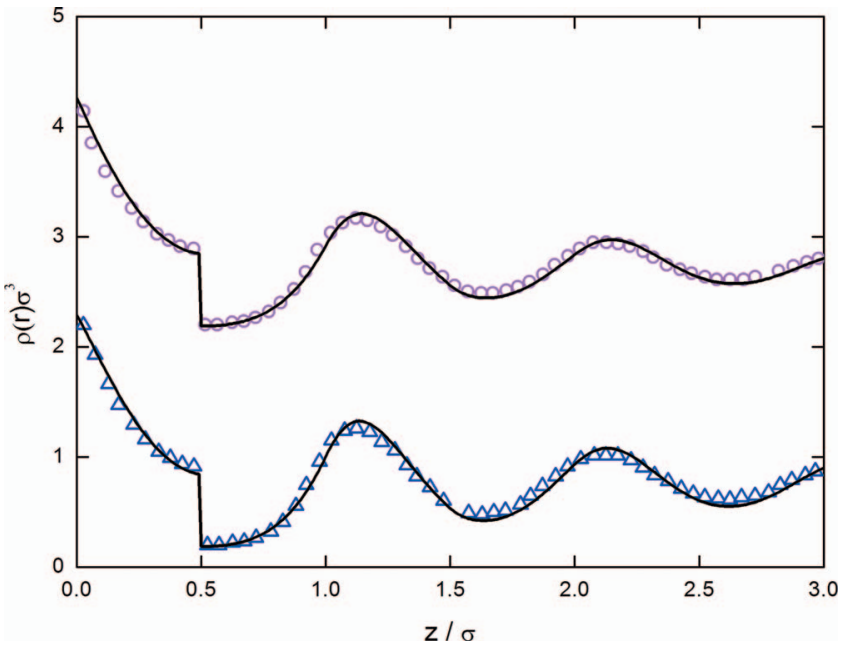

FIG. 1. Density profiles of SW fluid confined in an attractive slit. Curves: DFT model predictions; Symbols: MC simulation data, $\triangle$ : Case 3; $\bigcirc$ : Case 6 , as listed in Table I. The density profile of case 6 was shifted upward by 2.0 .

$\rho_{a v}$ is the average density in the pore $\left(=\frac{1}{H} \int_{0}^{H} d z \rho(z)\right)$. The effects of fluid segment number (chain length), fluid packing fraction, temperature, as well as the properties of solid surface (interaction between surface and fluid) on density profile were studied. The cases studied in this work are summarized in Table II, and Figures 2 and 3 illustrate the results for some cases. In all cases, $H=10 \sigma, \lambda=1.5$, and $\lambda_{\mathrm{w}}=1.0$.

From the model prediction and results shown in Figures 2 and 3, we can see that at low temperature and weak wall-segment attraction, the model captures the transition from depletion to adsorption as $\eta$ increases for short chain molecules, but for long chain molecules, the densities near the wall are low even at high $\eta$. At low temperature, the densities for short chain molecules near the wall increase significantly as the wall-segment attraction increases, and the densities for long chain molecules at high temperatures show the same (similar) results. The densities near the wall for long

TABLE II. Summary of the cases used for predicting the density profiles of square-well chain fluids in square-well slit pores.

\begin{tabular}{ccccc}
\hline \hline$m$ & $k T / \varepsilon_{f}$ & $\varepsilon_{w} / k T$ & $\eta$ & No. case \\
\hline 3 & 3 & 0.3 & 0.1 & 1 \\
& & 0.2 & 2 \\
& & 0.3 & 3 \\
& & & 0.1 & 4 \\
& & & 0.2 & 5 \\
& & & 0.3 & 6 \\
& \multirow{2}{*}{3} & & 0.1 & 7 \\
& & & 0.2 & 8 \\
& & & 0.3 & 9 \\
& & & 0.1 & 10 \\
& & & 0.2 & 11 \\
& & & 0.3 & 12 \\
& & & 0.1 & 13 \\
& & 0.2 & 14 \\
& & 0.3 & 15 \\
\hline \hline
\end{tabular}

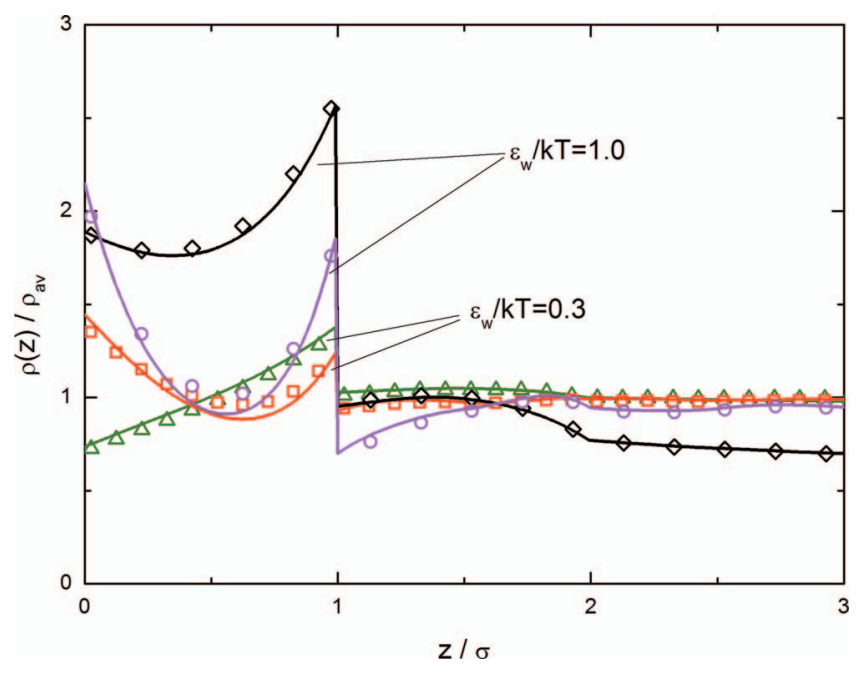

FIG. 2. Density profiles of 3-mer near one wall of the slit. Curves: DFT model predictions; Symbols: MC simulation data, $\triangle$ : Case $1 ; \square$ : Case $3 ; \diamond$ : Case 4; $\bigcirc$ : Case 6, as listed in Table II.

chain molecules at high $\eta$ are more sensitive to temperature than those at low $\eta$.

The model prediction was compared with the molecular simulation results. ${ }^{39}$ The comparison shows that the model prediction can capture the effects of packing fraction $\eta$, temperature T, chain-length, as well as the properties of surfaces on the density profile of confined fluids in slit pores. For longer chains at low temperature, the model prediction is not as good as those under other conditions, and other DFT models also show some discrepancies. ${ }^{39}$

\section{Density profiles of LJ fluids in LJ slit pores}

In the second version of PC-SAFT, the segments interact with a modified square-well potential. The efficient hard sphere diameter was calculated by Barker and Henderson's perturbation theory. The parameters of pure n-alkanes were determined by using Lennard-Jones attractive potential in the

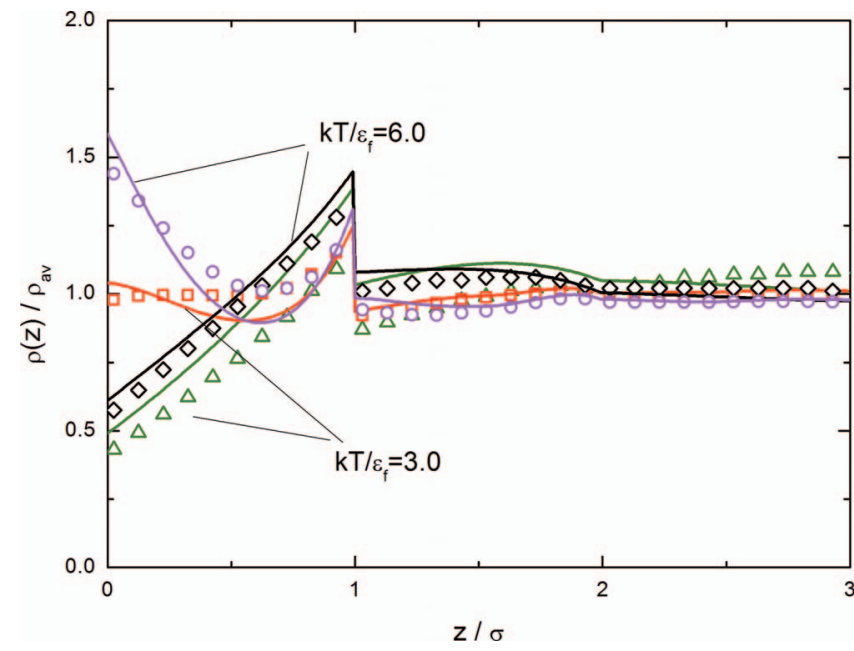

FIG. 3. Density profiles of 12-mer near one wall of the slit. Curves: DFT model predictions; Symbols: MC simulation data, $\triangle$ : Case $7 ; \square$ : Case $9 ; \diamond$ : Case 10; $\bigcirc$ : Case 12, as listed in Table II. 


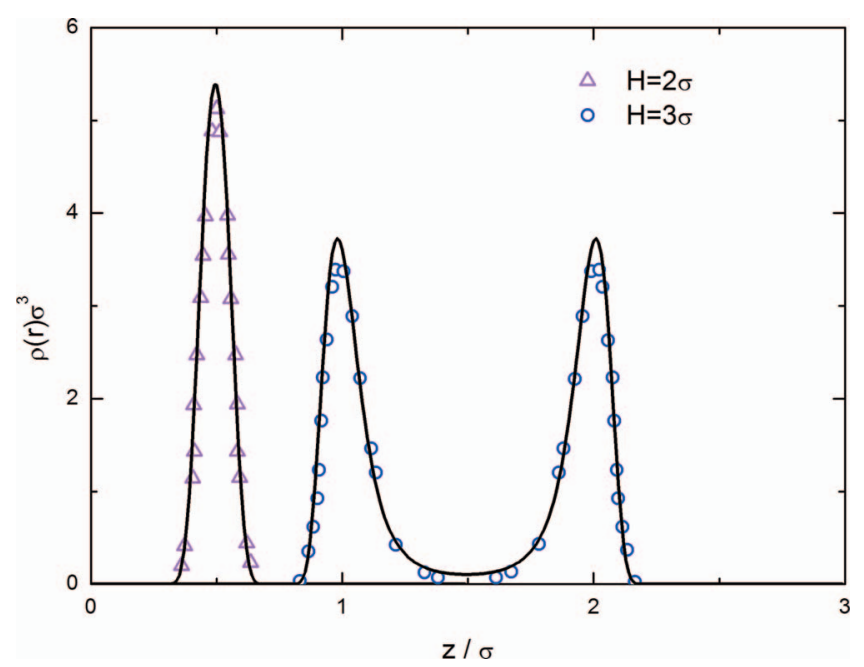

FIG. 4. Density profiles of LJ fluid confined in a slit. Curves: DFT model predictions; Symbols: simulation results: $\triangle: H=2 \sigma ; \bigcirc: H=3 \sigma$. The density profile of $H=2 \sigma$ was shifted left by 0.5 .

expression of the dispersion term; subsequently, the universal model constants in the dispersion term were regressed using the pure parameters of n-alkanes. Therefore, PC-SAFT is compatible with Lennard-Jones chain theory.

The DFT model in this work is based on the second version of PC-SAFT while the weighted density was calculated based on square-well fluid, and thus the developed model is not a theory for LJ chain. To further investigate the compatibility of the developed DFT model in this work with LJ fluids, we compared the density distribution predicted from the DFT model with the simulation results of LJ monomers and $\mathrm{LJ}$ chains confined in a slit. As mentioned in the forgoing text, the value of $\lambda$ was set to be 1.5 in the developed model in this section.

The density profiles of LJ monomers confined in a slit pore with width $H$ of $2 \sigma, 3 \sigma$, and $7.5 \sigma$, respectively, were studied using the developed DFT model. The interaction between the surface of the slit and $\mathrm{LJ}$ fluid was given by ${ }^{40}$

$$
V_{\text {ext }}(z)=\varepsilon_{w}\left[\frac{2}{5}\left(\frac{\sigma_{w}}{z}\right)^{10}-\left(\frac{\sigma_{w}}{z}\right)^{4}-\frac{\sigma_{w}^{4}}{3 \Delta(z+0.61 \Delta)^{3}}\right],
$$

where $\Delta$ was set to be $\sigma / \sqrt{2}, \sigma_{w}$ was set to be $\sigma$, and $\varepsilon_{w}$ was set to be $2 \pi \varepsilon_{f} .^{40}$ This potential was widely used for modeling of interaction between fluid molecule and planar surface. $\sigma_{w}$ and $\varepsilon_{w}$ are the energy and diameter parameter for solid-fluid interaction, and $\Delta$ is the interlayer distance.

The bulk density $\left(\rho_{b} \sigma^{3}\right)$ was set to be 0.5952 , and $k T / \varepsilon_{f}$ was set to be 1.2. The model prediction is illustrated in Figures 4 and 5 where the molecule simulation results ${ }^{40}$ are depicted as symbols for comparison. For all the cases studied in this section, the model prediction shows excellent agreement with simulation results.

We further considered four LJ chains (monomer, 4-mer, 8-mer, 16-mer) confined in an attractive slit, and the external field was described by Eq. (19) with $\Delta=0.7071 \sigma, \sigma_{w}=\sigma$, $\varepsilon_{w}=6.283 \varepsilon_{f} .{ }^{40}$ The average packing fraction of chains inside the slit was fixed at $\eta_{a v}=0.1$.

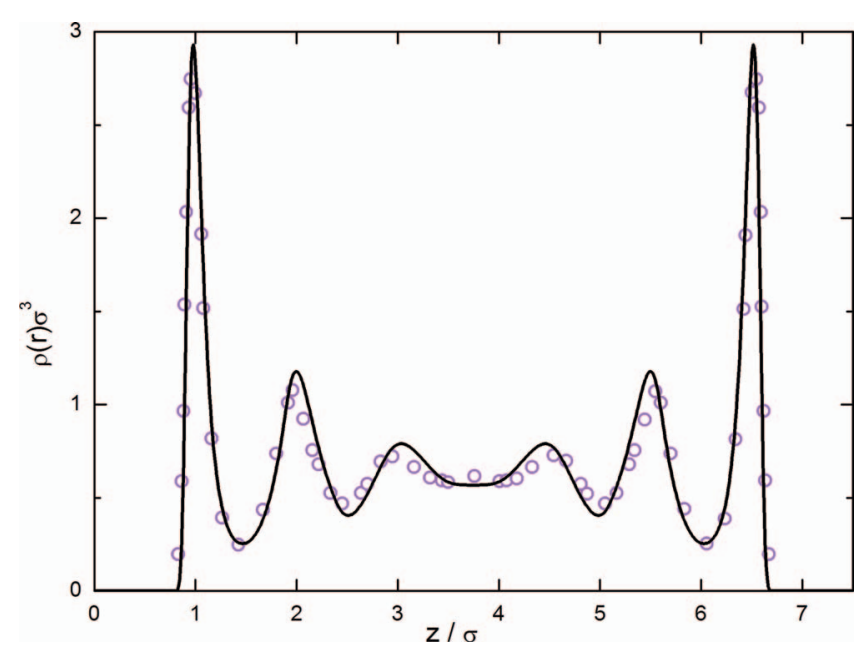

FIG. 5. Density profile of LJ fluid confined in a slit with width $H=7.5 \sigma$. Curve: DFT model prediction; Symbols: simulation results.

Figure 6 displays the segment density profiles $\left(\rho_{\text {seg }}(\boldsymbol{r})\right.$ $=m \rho(\boldsymbol{r}))$ of LJ chains near a surface of the slit. Results of monomer and long chains predicted by the theory are in good agreement with simulation data. ${ }^{41}$ At this temperature, the density at the first peak increases with increasing chain length, and the density is nearly zero away from the surface.

The agreement of the model prediction with the molecule simulation results for LJ fluids reveals that the PC-SAFT-DFT is compatible with LJ-based theory although the PC-SAFT model is based on modified square-well fluids and the developed DFT model is not a theory for LJ fluids.

\section{Adsorption isotherm representation}

In Secs. III A-III C, the prediction of the developed model was verified by comparing with the molecular simulation results, and the agreement indicates the model's reliability. As shown in Secs. III A-III C, the substance studied

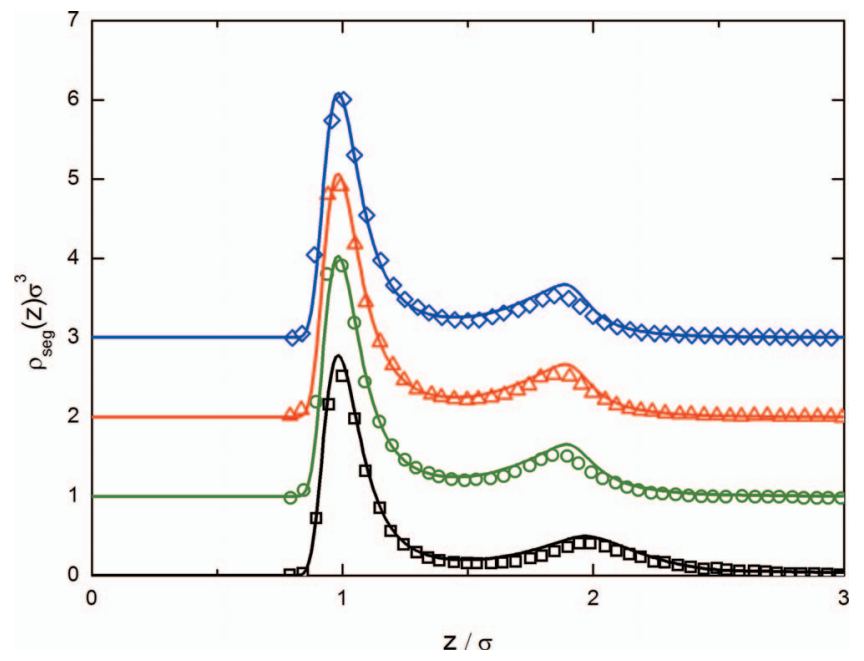

FIG. 6. Segment density profiles for LJ chains in an attractive slit, with the width of the slit $H=10 \sigma$. The reduced temperature is $k T / \varepsilon_{f}=1.0$. The symbols represent the simulation data: $\square$ : 1-mer; $\bigcirc$ : 4-mer; $\triangle$ : 8-mer; $\diamond$ : 16-mer. The density profiles were shifted upward for chains by $1.0,2.0$, and 3.0 , respectively. 
is "model molecule," and the segment number for the chain molecule is always an integer. However, for a physically existing substance, it can be modeled as a chain molecule in PC-SAFT, and the segment number is not necessarily an integer. For a long chain molecule, neglecting the decimal part may not affect the model results so much, for example, when the segment number is more than 100 , and it is reasonable to set the segment number as an integer. However, for a smaller chain molecule, the segment number of PC-SAFT obtained from fluid bulk properties is a non-integer for most real substances, and neglecting the decimal part will affect the model results significantly. Therefore, it is important to investigate the model performance for a real substance, especially for the small molecules.

The gas adsorption of small molecules such as methane $\left(\mathrm{CH}_{4}\right)$ and carbon dioxide $\left(\mathrm{CO}_{2}\right)$ on nanoporous materials has been widely studied experimentally, and the adsorption isotherm has been measured extensively for the purpose of separating $\mathrm{CO}_{2}$ from gas mixture or selective exchange adsorption of $\mathrm{CO}_{2}$ from $\mathrm{CH}_{4}$ for enhanced coal-bed methane. To illustrate the developed DFT model performance, the adsorption of methane and carbon dioxide on Calgon F400 activated carbon was studied as an example in this work.

In general, the material has a wide range of pore size distribution and shape. In order to reduce the computation complexity, in modeling, we assumed that the gas molecule was adsorbed in a single slit-like pore with an average width. The solid-fluid interaction $V_{\text {ext }}(z)$ was represented by Lee's $10-4$ Lennard-Jones potential, ${ }^{42}$ which is given by

$$
\begin{aligned}
V_{\text {ext }}(z)= & 2 \pi \rho_{\text {atom }} \varepsilon_{s f} \sigma_{s f}^{2} \\
& \times\left[\frac{2}{5}\left(\frac{\sigma_{s f}}{z}\right)^{10}-\sum_{i=1}^{4} \frac{\sigma_{s f}^{4}}{\left(z+(i-1) \sigma_{s}\right)^{4}}\right],
\end{aligned}
$$

where $\rho_{\text {atom }}$ is the solid atom density, $\sigma_{s f}$ is the solid-fluid diameter, $\sigma_{s}$ is the carbon interplanar distance, and $\varepsilon_{s f}$ is the potential representing the interaction between surface and fluid segment.

The excess adsorption $(\Gamma)$ of gas is calculated by

$$
\Gamma=\frac{A^{*}}{2} \int_{\sigma_{s f}}^{H-\sigma_{s f}} d z\left(\rho(z)-\rho_{b u l k}\right),
$$

where $A^{*}$ is the surface area of the adsorbent, and $H$ is the width of the slit.

To represent gas adsorption, the properties of fluid and solid surface need to be provided. In this work, the parameters of fluids were taken from Ref. 19, which were obtained from the fitting of the experimental data in bulk phase. The parameters for $\mathrm{CH}_{4}$ and $\mathrm{CO}_{2}$ used in this work are listed in Table III.

TABLE III. Molecular parameters. ${ }^{19}$

\begin{tabular}{llll}
\hline \hline Substance & \multicolumn{1}{c}{$m$} & $\sigma_{f}(\AA)$ & $\varepsilon_{f} / k(\mathrm{~K})$ \\
\hline $\mathrm{CH}_{4}$ & 1.0 & 3.7039 & 150.03 \\
$\mathrm{CO}_{2}$ & 2.0729 & 2.7852 & 169.21 \\
\hline \hline
\end{tabular}

The parameters of solid (the wall) can be adjustable or estimated. In order to decrease the number of adjustable parameters, in this work, some parameters of solid were taken from Ref. 42, i.e., for Calgon F400 activated carbon, the solid atom density $\rho_{\text {atom }}$ was set to be 0.382 atoms $/ \AA^{2}$, and $\sigma_{s}$ was set to be $3.35 \AA$. In addition, the following mixing rule was used to obtain $\sigma_{s f}$,

$$
\sigma_{s f}=\frac{\sigma_{s}+\sigma_{f}}{2} .
$$

The surface area of the adsorbent was taken from the experimental BET (Brunauer-Emmett-Teller) surface area in this work. For Calgon F400 activated carbon, $A^{*}$ is $850 \mathrm{~m}^{2} / \mathrm{g} \cdot{ }^{43}$

The adsorption quantity calculated by the model is sensitive to the value of slit width $H$. As $H$ was assumed to be the average pore width, in this work $H$ was considered an adjustable parameter but set to be a fixed value for the same porous materials, and then it is independent of the adsorbed gas. $\varepsilon_{s f}$ was set as one more adjustable parameter for one system. Therefore, in this work, there are three adjustable parameters in total in order to represent the adsorption of methane and carbon dioxide on Calgon F400 activated carbon, i.e., the size of pore $H$, and two LJ interaction parameters $\varepsilon_{s f}$ for two systems. These three adjustable parameters were obtained from the fitting of experimental data of gas adsorption isotherms for both $\mathrm{CH}_{4}$ and $\mathrm{CO}_{2}$ on Calgon $\mathrm{F} 400$ activated carbon with the following objective function,

$$
O b j=\sum_{i=1}^{N^{\exp }}\left|\frac{\Gamma_{i}^{c a l}-\Gamma_{i}^{\exp }}{\Gamma_{i}^{\exp }}\right|^{2},
$$

where $N^{\exp }$ is the number of experimental data points, $\Gamma_{i}^{c a l}$ is the calculated excess adsorption value, and $\Gamma_{i}^{\text {exp }}$ is the experimental excess adsorption value. The average relative deviation $\left(A R D=\frac{100}{N^{\exp }} \sum_{i=1}^{N^{\text {exp }}}\left|\frac{\Gamma_{i}^{c a l}-\Gamma_{i}^{\text {exp }}}{\Gamma_{i}^{\text {exp }}}\right|\right)$ was used to illustrate the model performance.

Using the experimental data measured by Sudibandriyo and co-workers at $318.2 \mathrm{~K},{ }^{43}$ the DFT model parameters were obtained and listed in Table IV with the average relative deviation $6.17 \%$. Both the model results and experimental data are shown in Figures 7 and 8 with reasonable agreements, which implies that it is feasible to represent the adsorption of real substances with integer or non-integer segment numbers.

As the total adsorption was reported and studied in other works, using the model parameters, the total adsorption for these two substances on Calgon F400 activated carbon was calculated and compared with the experimental data too. The model performances on excess adsorption and total adsorption are similar, which implies that it is reasonable to obtain

TABLE IV. Regressed pure-component parameters.

\begin{tabular}{cccc}
\hline \hline & \multicolumn{3}{c}{$\varepsilon_{s f} / k(\mathrm{~K})$} \\
\cline { 2 - 3 }$(\AA)$ & $\mathrm{CH}_{4}$ & $\mathrm{CO}_{2}$ & ARD (\%) \\
\hline 13.22 & 76.9 & 67.06 & 6.17 \\
\hline \hline
\end{tabular}




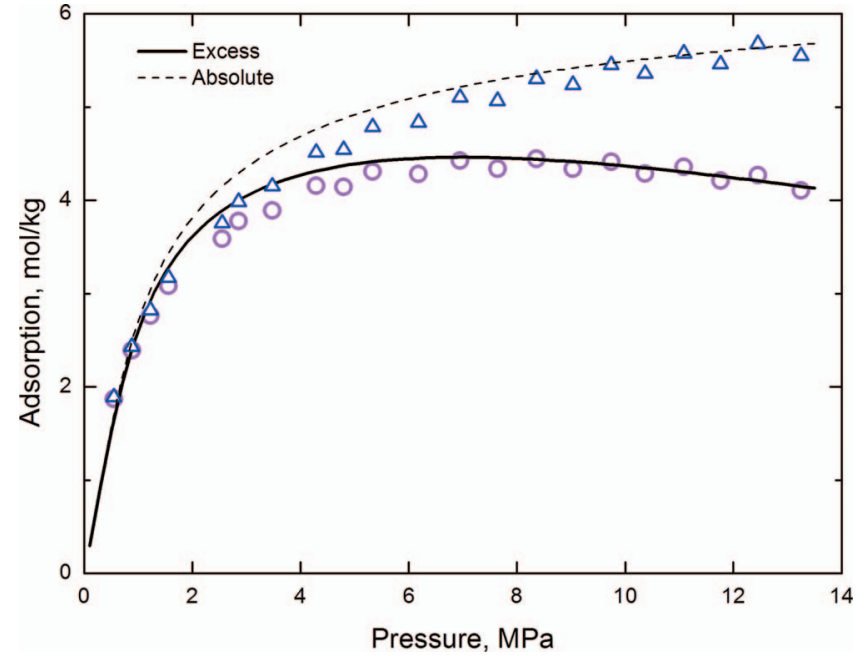

FIG. 7. Methane adsorption on Calgon F400 activated carbon. Symbols are experimental data; curves represent the results of the model.

the model parameters from the experimental data of excess adsorption.

The main focus of this section is to illustrate the model feasibility for representing the confined behaviors of real small substances with non-integer segment numbers, and the results reveal the model's feasibility as well as reasonable performance. To improve the model performance, more adjustable parameters can be set, which has been widely used in other models. However, this is not the focus of this work, and thus no more work was done here.

It should be noted that the DFT models developed by Yu and $\mathrm{Wu}^{8}$ and by $\mathrm{Ye}$ et al. ${ }^{10}$ can provide accurate descriptions of long chain polymers compared with simulation. However, in all these developed models, the segment number of the molecule can only be an integer, which limits their application to real substances. The other developed DFT model has been used to describe the interfacial tension, but their application to confined fluids has not been available. The developed model in this work is completely consistent with bulk PC-SAFT, and

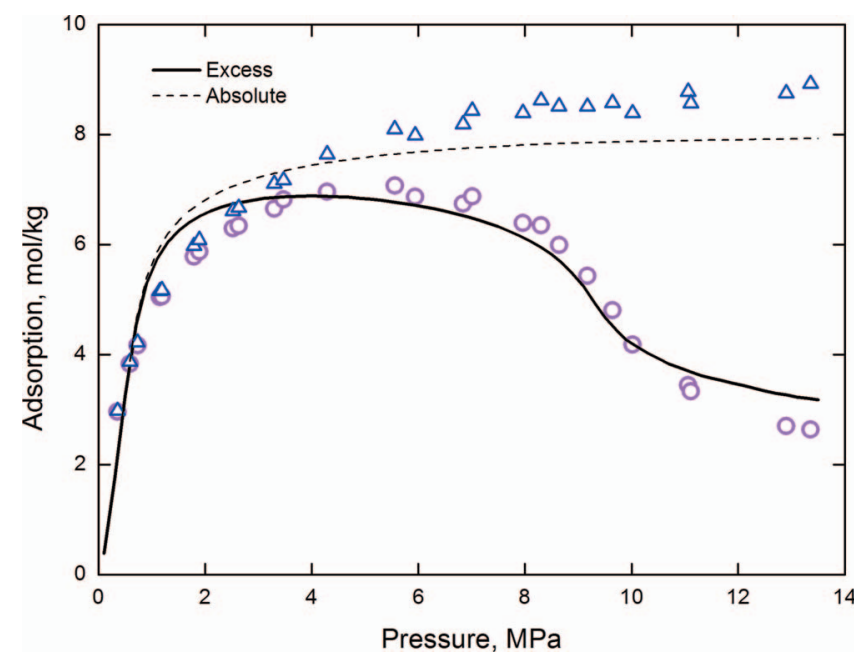

FIG. 8. Carbon dioxide adsorption on Calgon F400 activated carbon. Symbols are experimental data; curves represent the results of the model. its successful application in real substances shows the feasibility to represent the properties of fluids from homogeneous and inhomogeneous fields with the same set of parameters.

\section{CONCLUSIONS}

In this work, we developed a density functional theory that is consistent with the bulk PC-SAFT model. We predicted the density distributions of square-well chains confined in attractive slit and compared them with simulation results. The comparison shows that the DFT model provides sufficient accurate density distribution predictions. As PC-SAFT is compatible with Lennard-Jones molecules in bulk, we also compared the density profiles predicted from the DFT model with the simulation results for Lennard-Jones chains confined in an attractive slit, and again the DFT model prediction is in good agreement with simulation results. Furthermore, the DFT model developed in this work was used to represent the isotherm adsorption of methane and carbon dioxide on activated carbons. The pore of the activated carbon was modeled as a slit with a Lennard-Jones potential interacting between slit surface and fluid. The parameters of methane and carbon dioxide were the same as those in the bulk PC-SAFT. The study illustrates the model's feasibility for representing the confined behaviors of physically existing small substances with non-integer segment numbers.

\section{ACKNOWLEDGMENTS}

X.J. thanks Swedish Research Council, and financial support from European Commission under EU FP7, Grant No. NMP4-SL-2012-280519, NanoSelect, is gratefully acknowledged. G.S. and X.L. thank the Key Project of the National Natural Science Foundation of China (Grant No. 21136004), and National Basic Research Program of China (Grant No. 2013CB733500). X.J. thanks Professor Gross at Universität Stuttgart for his valuable comments and discussions.

${ }^{1}$ J. T. Eijkel and A. Berg, Microfluid. Nanofluid. 1, 249-267 (2005).

${ }^{2}$ E. Hendriks, G. M. Kontogeorgis, R. Dohrn, J.-C. de Hemptinne, I. G. Economou, L. F. Žilnik, and V. Vesovic, Ind. Eng. Chem. Res. 49, 1113111141 (2010).

${ }^{3}$ D.-E. Jiang, Z. Jin, and J. Wu, Nano Lett. 11, 5373-5377 (2011).

${ }^{4}$ T. Keshavarzi, F. Sedaghat, and G. A. Mansoori, Microfluid. Nanofluid. 8, 97-104 (2010).

${ }^{5}$ K. P. Travis and K. E. Gubbins, J. Chem. Phys. 112, 1984-1994 (2000).

${ }^{6}$ J. Z. Wu, AIChE J. 52, 1169-1193 (2006).

${ }^{7}$ Y. X. Yu and J. Z. Wu, J. Chem. Phys. 116, 7094-7103 (2002).

${ }^{8}$ Y. X. Yu and J. Z. Wu, J. Chem. Phys. 117, 2368-2376 (2002).

${ }^{9}$ S. Tripathi and W. G. Chapman, J. Chem. Phys. 122, 094506 (2005).

${ }^{10}$ Z. C. Ye, J. Cai, H. L. Liu, and Y. Hu, J. Chem. Phys. 123, 194902 (2005).

${ }^{11}$ C. P. Emborsky, Z. Feng, K. R. Cox, and W. G. Chapman, Fluid Phase Equilib. 306, 15-30 (2011).

${ }^{12}$ W. G. Chapman, K. E. Gubbins, G. Jackson, and M. Radosz, Ind. Eng. Chem. Res. 29, 1709-1721 (1990).

${ }^{13}$ A. Gil-Villegas, A. Galindo, P. J. Whitehead, S. J. Mills, G. Jackson, and A. N. Burgess, J. Chem. Phys. 106, 4168-4186 (1997).

${ }^{14}$ F. J. Blas and L. F. Vega, Mol. Phys. 92, 135-150 (1997).

${ }^{15}$ H. Adidharma and M. Radosz, Ind. Eng. Chem. Res. 37, 4453-4462 (1998).

${ }^{16}$ X. Y. Ji, S. P. Tan, H. Adidharma, and M. Radosz, Ind. Eng. Chem. Res. 44, 7584-7590 (2005).

${ }^{17}$ S. P. Tan, H. Adidharma, and M. Radosz, Ind. Eng. Chem. Res. 44, 44424452 (2005) 
${ }^{18}$ J. Gross and G. Sadowski, Fluid Phase Equilib. 168, 183-199 (2000).

${ }^{19}$ J. Gross and G. Sadowski, Ind. Eng. Chem. Res. 40, 1244-1260 (2001).

${ }^{20}$ S. P. Tan, H. Adidharma, and M. Radosz, Ind. Eng. Chem. Res. 47, 80638082 (2008).

${ }^{21}$ E. A. Muller and K. E. Gubbins, Ind. Eng. Chem. Res. 40, 2193-2211 (2001).

${ }^{22}$ I. G. Economou, Ind. Eng. Chem. Res. 41(5), 953-962 (2002).

${ }^{23}$ G. J. Gloor, F. J. Blas, E. M. del Río, E. de Miguel, and G. Jackson, Fluid Phase Equilib. 194-197, 521-530 (2002).

${ }^{24}$ G. J. Gloor, G. Jackson, F. J. Blas, E. M. del Río, and E. de Miguel, J. Chem. Phys. 121, 12740-12759 (2004).

${ }^{25}$ G. J. Gloor, G. Jackson, F. J. Blas, E. M. del Río, and E. de Miguel, J. Phys. Chem. C 111, 15513-15522 (2007).

${ }^{26}$ J. Gross, J. Chem. Phys. 131, 204705 (2009).

${ }^{27}$ S. Tripathi and W. G. Chapman, Phys. Rev. Lett. 94, 087801 (2005).

${ }^{28}$ Y. X. Yu and J. Z. Wu, J. Chem. Phys. 117, 10156-10164 (2002).

${ }^{29}$ T. Boublik, J. Chem. Phys. 53, 471-472 (1970).

${ }^{30}$ G. A. Mansoori, N. F. Carnahan, K. E. Starling, and J. T. W. Leland, J. Chem. Phys. 54, 1523-1525 (1971).
${ }^{31}$ Y. Rosenfeld, Phys. Rev. Lett. 63, 980 (1989).

${ }^{32}$ J. Y. Hu, J. Chen, and J. G. Mi, Ind. Eng. Chem. Res. 51(3), 1236-1243 (2012).

${ }^{33}$ B. Peng and Y. X. Yu, Langmuir 24, 12431-12439 (2008).

${ }^{34}$ S. P. Hlushak, C. McCabe, and P. T. Cummings, J. Chem. Phys. 137, 104104 (2012)

${ }^{35}$ S.-C. Kim and S. H. Lee, J. Phys.: Condens. Matter 16, 6365 (2004).

${ }^{36}$ S. Q. Zhou and J. R. Solana, Chem. Rev. 109(6), 2829-2858 (2009).

${ }^{37}$ S. P. Tan, H. Adidharma, and M. Radosz, Ind. Eng. Chem. Res. 43, 203208 (2004).

${ }^{38}$ Z. H. Jin, Y. P. Tang, and J. Z. Wu, J. Chem. Phys. 134, 174702 (2011).

${ }^{39}$ Z. C. Ye, Ph.D. dissertation, East China University of Science and Technology, 2005.

${ }^{40}$ Y. X. Yu, J. Chem. Phys. 131, 024704 (2009).

${ }^{41}$ Z. D. Li, D. P. Cao, and J. Z. Wu, J. Chem. Phys. 122, 224701 (2005).

${ }^{42}$ S. A. Mohammad, A. Arumugam, R. L. Robinson, and K. A. M. Gasem, Energy Fuels 26, 536-548 (2012).

${ }^{43}$ M. Sudibandriyo, Z. Pan, J. E. Fitzgerald, R. L. Robinson, and K. A. M. Gasem, Langmuir 19, 5323-5331 (2003). 